\title{
Follicle-stimulating hormone receptor gene polymorphism and ovarian responses to controlled ovarian hyperstimulation for IVF-ET
}

\author{
Jong Kwan Jun · Ji Sung Yoon · Seung-Yup Ku $\cdot$ Young Min Choi · \\ Kyu Ri Hwang · Seo Young Park · Gyoung Hoon Lee · Won Don Lee • \\ Seok Hyun Kim · Jung Gu Kim · Shin Yong Moon
}

Received: 11 March 2006/ Accepted: 22 April 2006/ Published online: 27 July 2006

(C) The Japan Society of Human Genetics and Springer-Verlag 2006

\begin{abstract}
This study was performed to investigate the association between FSH receptor (FSHR) gene polymorphism at position 680 and the outcomes of controlled ovarian hyperstimulation $(\mathrm{COH})$ for in vitro fertilization and embryo transfer (IVF-ET) in Korean women. Two hundred and sixty-three patients under 40 years of age who underwent IVF-ET procedures were included in this study. Patients with polycystic ovary syndrome, endometriosis, or a previous history of ovarian surgery were excluded. Following extraction of genomic DNA, the FSHR polymorphism at position 680 was determined by polymerase chain reaction and restriction fragment length polymorphism analysis. The FSHR genotype distribution was $41.8 \%$ for Asn/ Asn, $45.6 \%$ for Asn/Ser, and $12.5 \%$ for Ser/Ser FSHR genotype groups. Although there was no difference among the three genotype groups in terms of the age and infertility diagnosis of study subjects, the basal levels of FSH (day 3) were significantly different $[5.7 \pm 0.3 \mathrm{IU} / 1 \quad($ mean $\pm \mathrm{SEM}), \quad 6.0 \pm 0.3 \mathrm{IU} / 1, \quad$ and $8.2 \pm 0.9 \mathrm{IU} / 1$ for Asn/Asn, Asn/Ser, and Ser/Ser groups, respectively. The Ser/Ser group tended to require a higher dose of gonadotropins for $\mathrm{COH}$, and
\end{abstract}

J. K. Jun · S.-Y. Ku · Y. M. Choi $(\bowtie) \cdot$ K. R. Hwang

G. H. Lee - S. H. Kim · J. G. Kim - S. Y. Moon

Department of Obstetrics and Gynecology,

The Institute of Reproductive Medicine and Population,

Medical Research Center, Seoul National University

College of Medicine, 28 Yungun-dong, Chongno-ku,

Seoul 110-744, South Korea

e-mail: ymchoi@snu.ac.kr

J. S. Yoon · S. Y. Park · W. D. Lee

Department of Obstetrics and Gynecology,

Maria Infatility Hospital, Seoul, South Korea tended to show lower serum estradiol levels at the time of hCG administration than the other two groups, though these differences did not reach statistical significance. The numbers of oocytes retrieved tended to be different for the three groups $(9.6 \pm 0.6,10.2 \pm 0.6$, and $7.9 \pm 0.8$ for Asn/Asn, Asn/Ser, and Ser/Ser groups, respectively). Clinical pregnancy rate was significantly higher in Asn/Asn, compared to the others (45.7 vs. $30.5 \%, P=0.013$ ). The homozygous Ser/Ser genotype of FSHR polymorphism at position 680 may be associated with a reduced ovarian response to $\mathrm{COH}$ for IVF-ET, while Asn/Asn genotypes showed a higher pregnancy rate.

Keywords FSH receptor · Polymorphism - IVF-ET · Controlled ovarian hyperstimulation $(\mathrm{COH})$

\section{Introduction}

Follicle-stimulating hormone (FSH) plays a major role during folliculogenesis, and its action is mediated by FSH receptor (FSHR) (Fauser and van Heusden 1997). Urinary and recombinant FSH are major gonadotropins that have been widely used for controlled ovarian hyperstimulation $(\mathrm{COH})$ in assisted reproductive technology (ART). Despite attempts to standardize $\mathrm{COH}$ regimens for women undergoing ART procedures, we commonly experience either low ovarian responses or ovarian hyperstimulation syndrome (OHSS) even in those with similar endocrine profiles.

This individual variability in ovarian response necessitates the identification of predictive markers of ovarian hyper- or hyporesponse of corresponding $\mathrm{COH}$ cycles, which would be of a clinical value in ART 
programs (Kligman and Rosenwaks 2001). Of the various predictors of ovarian reserve, basal FSH level is considered the most useful one in clinical practice (Balasch et al. 1996); however, basal FSH varies from cycle to cycle in the same individual (Tinkanen et al. 1999).

In animal studies, it is known that ovarian response to gonadotropin stimulation is influenced by genetic factors (Spearow and Barkley 1999). Regarding the ligand, many researchers have attempted to evaluate differences in the distribution of FSH isoforms, to identify the presence of inhibitors or antibodies that are combined with FSH, and to characterize the different signals caused by the various FSH isoforms (Fauser and van Heusden 1997; Ulloa-Aguirre et al. 1995; Fauser 1996). However, few reports have demonstrated the efficacy of measuring FSH isoforms for predicting the prognosis of ART cycles.

FSH receptors are expressed on the granulosa cells of ovarian follicles. They are composed of seven transmembrane domains that are connected by three intra- and extracellular chains (Simoni et al. 1997). Recent studies have tried to evaluate the distribution of various FSHR gene polymorphisms among infertile patients. At present, three polymorphisms of FSHR gene have been identified: one in the promoter region and two in exon 10 . The polymorphisms at positions 307 and 680 in exon 10 are in strong linkage disequilibrium, yielding two common variants: $\mathrm{Thr}^{307}-\mathrm{Asn}^{680}$ and $\mathrm{Ala}^{307}-\mathrm{Ser}^{680}$. Moreover, a recent study reported that FSHR gene polymorphism at position 680 is an important prognosticator of ovarian response to FSH stimulation (Mayorga et al. 2000).

It would be of substantial clinical value if this polymorphism of FSHR gene could be used to predict ovarian response prior to starting $\mathrm{COH}$ cycles. At present, no report is available on this polymorphism in infertile Asian women. Thus, in this study, we sought to investigate the distribution of FSHR gene polymorphism in infertile Korean women and to evaluate its impact on the results of $\mathrm{COH}$ and pregnancy in in vitro fertilization and embryo transfer (IVF-ET) cycles.

\section{Materials and methods}

\section{Subjects}

Two hundred and sixty-three women who underwent an IVF-ET procedure were prospectively recruited for this study. All were younger than 40 years of age and underwent the procedure in response to tubal, male or unexplained infertility. Patients with polycystic ovary syndrome, endometriosis, or a previous history of ovarian surgery were excluded. The institutional review board for human research at Seoul National University Hospital approved this project, and written informed consent was obtained from all study subjects.

\section{$\mathrm{COH}$ and IVF-ET protocols}

Highly purified FSH (Metrodin-HP, Serono, Switzerland) or recombinant FSH (Gonal-F, Serono, Switzerland) was used for $\mathrm{COH}$. Stimulation was performed using either GnRH agonist long (83 women, $31.6 \%)$ or $\mathrm{GnRH}$ antagonist multidose protocol (180 women, $68.4 \%$ ).

For GnRH agonist long protocols, daily treatment with $0.1 \mathrm{mg}$ triptorelin (Decapeptyl, Ferring, Sweden) began on day 21 of the preceding cycle of stimulation cycle, and continued until the day of hCG treatment. Treatment with two to four ampules of FSH, depending on the patients' previous or anticipated responses, was initiated on day 3 of the menstrual cycle. Treatment was then individualized in a step-down fashion. For GnRH antagonist protocols, gonadotropins were started on day 3 and administered in a similar fashion to the GnRH agonist long protocol. A daily dose of $0.25 \mathrm{mg}$ cetrorelix (Cetrotide, Serono, Switzerland) was administered from the time the largest follicle reached $14 \mathrm{~mm}$ in diameter until hCG day.

When the leading follicle reached $18 \mathrm{~mm}$ in mean diameter with a serum estradiol $\left(E_{2}\right)$ level of $200 \mathrm{pg} / \mathrm{ml}$ per mature follicle, 10,000 U of hCG (Profasi, Serono, Switzerland) was administered. Oocyte retrieval was performed $36 \mathrm{~h}$ after the hCG injection. The ICSI (intracytoplasmic sperm injection) was performed according to conventional protocols and only on the MII oocytes. Up to four embryos were transferred on the second or third day after retrieval. For the assessment of embryo quality, we defined embryos as high quality embryos if they had at least six blastomeres and $<20 \%$ anucleate fragments and no apparent morphologic abnormalities. Embryos showing blastomere multinucleation, poor cell adhesion, uneven cell division and cytoplasmic abnormalities were defined as low quality. The luteal phase was supported by i.m. progesterone (Progest, Samil Pharm, Korea).

Analysis of FSHR gene polymorphism

Genomic DNA was extracted from peripheral blood using a Wizard DNA extraction kit (Promega, USA). The FSHR polymorphism at position 680 was determined by polymerase chain reaction (PCR) and restriction fragment length polymorphism (RFLP) 
Table 1 Distribution of FSHR genotypes by infertility factors

\begin{tabular}{lrrlr}
\hline & Asn/Asn & \multicolumn{1}{l}{ Asn/Ser } & \multicolumn{1}{l}{ Ser/Ser } & Total \\
\hline Tubal factors & $38(45.2 \%)$ & $35(41.7 \%)$ & $11(13.1 \%)$ & 84 \\
Male factors & $35(46.7 \%)$ & $34(45.3 \%)$ & $6(8.0 \%)$ & 75 \\
Tubal and & $5(35.7 \%)$ & $8(57.1 \%)$ & $1(7.1 \%)$ & 14 \\
$\quad$ male factors & & & & \\
$\begin{array}{l}\text { Unexplained } \\
\text { All patients }\end{array}$ & $32(35.6 \%)$ & $43(47.8 \%)$ & $15(16.7 \%)$ & 90 \\
& $110(41.8 \%)$ & $120(45.6 \%)$ & $33(12.5 \%)$ & 263 \\
\hline
\end{tabular}

analysis. PCR reaction mixture contained $0.1 \mu \mathrm{g}$ of genomic DNA, $0.4 \mu \mathrm{M}$ of each primer (upstream 5'TTTGTGGTCATCTGTGGCTGC3', downstream 5'C AAAGGCAAGGACTGAATTATC ATT3'), $1.25 \mathrm{U}$ of Taq polymerase, $1.5 \mathrm{mM}$ of $\mathrm{MgCl}_{2}$, and $200 \mu \mathrm{M}$ of dNTP. Following an initial denaturation step at $94^{\circ} \mathrm{C}$ for $5 \mathrm{~min}$, samples were subjected to 30 rounds of PCR at $94^{\circ} \mathrm{C}$ for $30 \mathrm{~s}, 60^{\circ} \mathrm{C}$ for $30 \mathrm{~s}$, and $72^{\circ} \mathrm{C}$ for $30 \mathrm{~s}$ with a final extension time of $10 \mathrm{~min}$ at $72^{\circ} \mathrm{C}$. The PCR products were digested with 2 IU of restriction enzyme BsrI (New England Biolabs, USA) at $65^{\circ} \mathrm{C}$ overnight, separated by $3 \%$ agarose gel electrophoresis, and identified using ethidium bromide staining.

Absence of BsrI cleavage indicated the presence of asparagines (Asn), and cleaved fragments at BsrI site indicated the presence of serine (Ser). Uncleaved bands indicated the asparagine homozygote (Asn/ Asn), cleaved bands the serine homozygote (Ser/Ser), and simultaneous cleaved and uncleaved bands the heterozygote (Asn/Ser).

Statistical analysis

Clinical data were compared among different genotypes by one-way ANOVA and chi-square test where appropriate using SPSS 11.0.1 package. A $P$ value of less than 0.05 was regarded as statistically significant.

\section{Results}

The frequencies of the FSHR genotypes at position 680 among subjects were as follows: Asn/Asn $41.8 \%$ $(n=110)$, Asn/Ser $45.6 \%(n=120)$, and Ser/Ser $12.5 \%$ $(n=33)$, respectively (Table 1$)$. Infertility factors were classified as tubal only, male only, both tubal and male, and unexplained. No significant difference was observed among the three groups in terms of the distribution of these infertility factors (Table 1).

The mean ages of the three genotype groups were similar, and a comparison of the stimulation protocols, which may have affected the individual ovarian response, also showed no differences between the three FSHR genotype groups (Table 2).

Day 3 basal FSH levels were significantly higher in the Ser/Ser group $(8.2 \pm 0.9 \mathrm{IU} / \mathrm{l}$, mean $\pm \mathrm{SEM})$ as compared to those from the Asn/Asn group (5.7 $\pm 0.3 \mathrm{IU} / \mathrm{l})$ or the Asn/Ser group $(6.0 \pm 0.3 \mathrm{IU} / \mathrm{l})(P=0.001) \quad(\mathrm{Ta}-$ ble 3).

The doses of gonadotrpins used and serum estradiol levels on the day of hCG administration were not significantly different among the three genotypes: Asn/ Asn 2,287 $\pm 65 \mathrm{IU}, \quad$ Asn/Ser 2,439 $\pm 60 \mathrm{IU}, \quad$ Ser/Ser $2,466 \pm 157 \mathrm{IU}(P=0.196) ;$ Asn/Asn $1,667 \pm 152 \mathrm{pg} / \mathrm{ml}$, Asn $/$ Ser $1,706 \pm 122 \mathrm{pg} / \mathrm{ml}$, Ser/Ser $1,199 \pm 167 \mathrm{pg} / \mathrm{ml}$ $(P=0.215)$ (Table 4). No difference was found between the three groups, either, in terms of fertilization rate, numbers of embryos transferred and high quality embryos transferred, and endometrial thickness at hCG day.

The number of oocytes retrieved tended to be different among FSHR genotype groups. The Ser/Ser

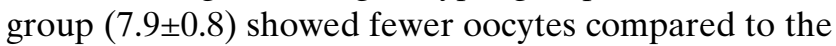
Asn/Ser group (9.6 \pm 0.6$)$. Clinical pregnancy rate per embryo transfer was significantly higher in the Asn/ Asn group (45.7\%) as compared to either the Asn/Ser group $(31.1 \%)$ or the Ser/Ser group $(28.1 \%)$. The $P$ value of this difference became smaller when the Asn/ Asn group was compared with the other two groups combined (45.7 vs. $30.5 \%, P=0.013$ ) (Table 4 ).

These similar findings were also shown when the outcomes were analyzed in GnRH agonist long protocol and GnRH antagonist protocol groups (Tables 5, 6). The differences in the dose of gonadotropins used and the number of oocytes retrieved were more remarkable when GnRH agonist was used. Pregnancy rate in the Asn/Asn group tended to be higher when

Table 2 Age of the patients and applied controlled ovarian hyperstimulation (COH) protocols for the three FSHR genotypes

\begin{tabular}{|c|c|c|c|c|c|}
\hline & Asn/Asn & Asn/Ser & Ser/Ser & Total & $P$ value \\
\hline Age & $32.1 \pm 0.3$ & $32.8 \pm 0.3$ & $32.9 \pm 0.5$ & $32.6 \pm 0.2$ & 0.213 \\
\hline \multicolumn{6}{|l|}{$\mathrm{COH}$ protocol } \\
\hline Antagonist protocol & $72(40.0 \%)$ & $87(48.3 \%)$ & $21(11.7 \%)$ & 180 & \\
\hline Long protocol & $38(45.8 \%)$ & $33(39.8 \%)$ & $12(14.5 \%)$ & 83 & \\
\hline All patients & $110(41.8 \%)$ & $120(45.6 \%)$ & $33(12.5 \%)$ & 263 & 0.423 \\
\hline
\end{tabular}

Values are mean \pm SEM 
Table 3 Basal FSH levels according to three FSHR genotypes

\begin{tabular}{lllll}
\hline & Asn/Asn & Asn/Ser & Ser/Ser & $P$ value \\
\hline Mean \pm SEM (IU/l) & $5.7 \pm 0.3$ & $6.0 \pm 0.3$ & $8.2 \pm 0.9$ & 0.001 \\
Median (IU/l) & 5.4 & 6.0 & 7.1 & \\
Range & $0.5-13.7$ & $0.3-14.5$ & $2.7-20.6$ & \\
\hline
\end{tabular}

Values are mean \pm SEM

GnRH agonist was used and was significantly higher when GnRH antagonist was used.

\section{Discussion}

Since the first report on the genotypic variance of the FSHR gene (Aittomaki et al. 1995), the possibility has been considered as to whether a single nucleotide polymorphism (SNP) of FSHR gene affects the ovarian response to exogenous gonadotropins. That is to say, the ovarian responses to gonadotropins for $\mathrm{COH}$ in IVF-ET cycles might differ according to FSHR gene genotypes.

Recently, Mayorga et al. (2000) evaluated the impacts of FSHR genotypes at position 680 on the ovarian responses to FSH stimulation in 161 infertile women undergoing IVF-ET. Although no differences were observed in terms of the number of oocytes retrieved and serum estradiol concentration on the day of hCG injection, the number of gonadotropin ampules and basal FSH levels were found to be higher in the Ser/Ser group. The researchers concluded that FSHR gene genotype is an important factor for determining the prognosis of $\mathrm{COH}$ cycles of normo-ovulatory infertile women.

In our study on infertile Asian women, FSHR gene polymorphism was found to differ from that of the
German women in the aforementioned study, which may be attributable to ethnicity. In particular, the frequency of the Ser/Ser genotype in our subjects was lower than that found in German women studied by Mayorga et al. (2000). However, the lower ovarian response in the Ser/Ser group, including high basal FSH and lower number of oocytes retrieved, is a similar finding.

The Ser/Ser genotype showed a tendency toward lower estradiol levels, lower numbers of oocytes and lower clinical pregnancy rates (Table 4). It may be assumed that differences in $\mathrm{COH}$ outcomes are related to a difference in FSHR genotype. It is expected that our findings would become more significant in further studies with a larger sample size.

Differences in the outcomes of IVF-ET according to FSHR genotypes were also observed when the outcomes were analyzed in GnRH agonist long protocol and GnRH antagonist protocol groups (Tables 5, 6). It could be inferred that the regimen of $\mathrm{COH}$ may not significantly affect the differences caused by different FSHR genotypes.

In regard to the mechanisms that result in these different IVF-ET outcomes, the authors are not sure about which factor-embryo quality, endometrial condition or luteal function-is responsible. Our study did not include an assessment of luteal function; however, there was no difference between the three groups in terms of fertilization rate, number of embryos and high quality embryos transferred, and endometrial thickness at hCG day (Table 4).

Sudo et al. (2002) first reported, in Asian women, on the role of FSHR gene polymorphism in ovarian response to $\mathrm{COH}$. However, the study analyzed only $\mathrm{COH}$ outcomes without pregnancy results in as few as 58 subjects. In addition, their study subjects included a

Table 4 Outcomes of controlled ovarian hyperstimulation according to FSHR genotypes

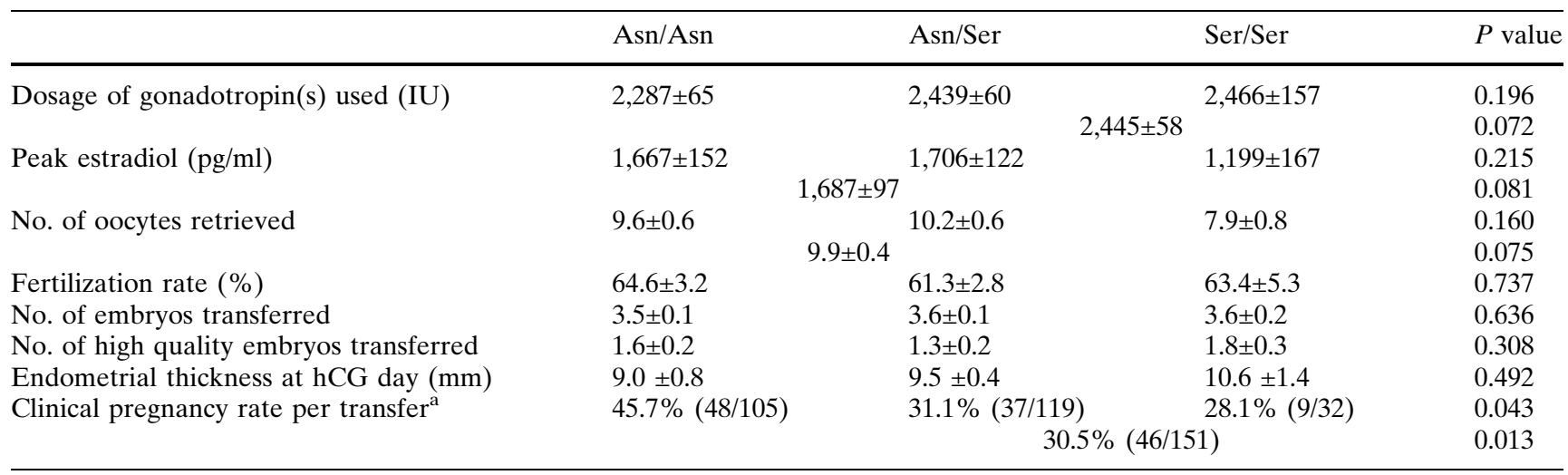

Values are mean \pm SEM. Values that span two columns are the mean of the individual values for the two groups combined

${ }^{\mathrm{a}}$ Embryo transfer was done in 256 patients 
Table 5 Outcomes of controlled ovarian hyperstimulation according to FSHR genotypes in patients in whom antagonist protocols were employed

\begin{tabular}{llcrr}
\hline & Asn/Asn & Asn/Ser & Ser/Ser & $P$ value \\
\hline Dosage of gonadotropin(s) used (IU) & $2,275 \pm 77$ & $2,375 \pm 61$ & $2,229 \pm 97$ & 0.440 \\
& & & $2,346 \pm 53$ & $1,172 \pm 169$ \\
Peak estradiol (pg/ml) & $1,800 \pm 194$ & & $1,660 \pm 149$ & 0.430 \\
& & $1,730 \pm 122$ & & 0.101 \\
No. of oocytes retrieved & $9.2 \pm 0.7$ & $9.7 \pm 0.6$ & 0.735 \\
Clinical pregnancy rate per transfer & & $9.5 \pm 0.5$ & $35 \%(7 / 20)$ & 0.577 \\
& $48.5 \%(33 / 68)$ & $31.0 \%(27 / 87)$ & $31.8 \%(34 / 107)$ & 0.026 \\
\hline
\end{tabular}

Values are mean \pm SEM. Values that span two columns are the mean of the individual values for the two groups combined

${ }^{a}$ Embryo transfer was done in 175 patients

Table 6 Outcomes of controlled ovarian hyperstimulation according to FSHR genotypes in patients in whom long protocols were employed

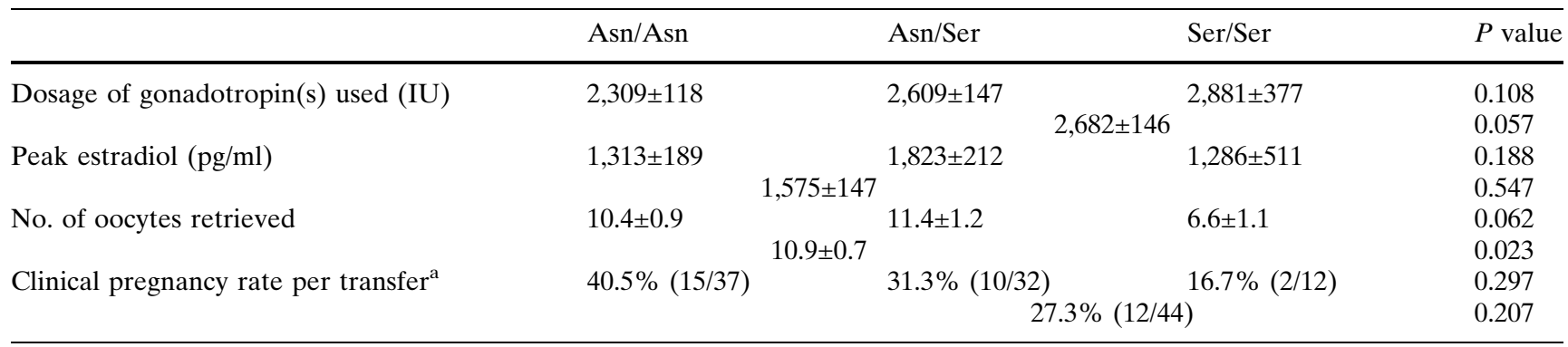

Values are mean \pm SEM. Values that span two columns are the mean of the individual values for the two groups combined

${ }^{a}$ Embryo transfer was done in 81 patients

significant number of women with polycystic ovary syndrome. Our data have probably shown, for the first time, a difference in pregnancy rate among different FSHR groups of infertile Asian women without evidence of polycystic ovary syndrome.

The efficacy of FSHR gene polymorphism in predicting poor response to $\mathrm{COH}$ has recently been reported (de Castro et al. 2003). This study also suggested the role of age within the same FSHR genotype. Significance was more remarkable in women $<35$ years compared with women $\geq 35$ years. The average age of our study subjects was less than 34 years in all three genotype groups. It may be expected that the differences found in our study will become less significant in older women.

The hypothesis underlying the present investigation was that different receptor genotypes might show different ovarian responses to exogenous gonadotropin administration. Several reports have provided possible explanations for molecular mechanisms that may support our hypothesis. Firstly, the expressions of cell surface receptors differ in the three genotypes, which would influence ligand/receptor interactions. More- over, such isoform differences might lead to different down regulation rates. Secondly, it should be considered that the individual receptor isoforms probably have different affinities for the different FSH isoforms (Zambrano et al. 1999). The presence of asparagine at position 680 is presumed to facilitate the expression of receptors via glycosylation process, whereas the presence of serine may accelerate phosphorylation and receptor turnover (Davis et al. 1995). These postulations indicate that minute genetic differences in the receptors may affect the action of the hormone at target cells.

In conclusion, this investigation reveals, in an Asian population, that FSHR gene polymorphism at position 680 may be associated with a different ovarian response to $\mathrm{COH}$. These findings should be confirmed in larger scale studies, which will probably reveal more significant results. In addition, further studies are necessary to determine whether it is possible to apply this relationship to the pre-cycle evaluation of individual genetic predisposition in terms of preventing either OHSS or a low ovarian response. 
Acknowledgements This study was supported by a grant of the Korea Health 21 R\&D Project, Ministry of Health and Welfare, Republic of Korea (01-PJ10-PG6-01GN13-0002).

\section{References}

Aittomaki K, Lucena JL, Pakarinen P, Sistonen P, Tapanainen J, Gromoll J, Kaskikari R, Sankila EM, Lehvaslaiho H, Engel AR et al (1995) Mutation in the follicle-stimulating hormone receptor gene causes hereditary hypergonadotropic ovarian failure. Cell 82:959-968

Balasch J, Creus M, Fabregues F, Carmona F, Casamitjana R, Ascaso C, Vanrell JA (1996) Inhibin, follicle-stimulating hormone, and age as predictors of ovarian response in in vitro fertilization cycles stimulated with gonadotropinreleasing hormone agonist-gonadotropin treatment. Am J Obstet Gynecol 175:1226-1230

de Castro F, Ruiz R, Montoro L, Perez-Hernandez D, SanchezCasas Padilla E, Real LM, Ruiz A (2003) Role of folliclestimulating hormone receptor Ser680Asn polymorphism in the efficacy of follicle-stimulating hormone. Fertil Steril 80:571-576

Davis D, Liu X, Segaloff DL (1995) Identification of the sites of $\mathrm{N}$-linked glycosylation on the follicle-stimulating hormone receptor and assessment of their role in FSH receptor function. Mol Endocrinol 9:159-170

Fauser BC (1996) Interference of follicle-stimulation hormone regulation of human ovarian function. Mol Hum Reprod 2:327-334
Fauser BC, van Heusden AM (1997) Manipulation of human ovarian function: physiological concepts and clinical consequences. Endocr Rev 18:71-106

Kligman I, Rosenwaks Z (2001) Differentiating clinical profiles: prediction good responders, poor responders, and hyperresponders. Fertil Steril 76:1185-1190

Mayorga MP, Gromoll J, Behre HM, Gassner C, Nieschlag E, Simoni M (2000) Ovarian response to follicle-stimulating hormone stimulation depends on the FSH receptor genotype. J Clin Endocrinol Metab 85:3365-3369

Simoni M, Gromoll J, Nieschalg E (1997) The follicle-stimulating hormone receptor: biochemistry, molecular biology, physiology, and pathophysiology. Endocr Rev 18:739-773

Spearow JL, Barkley M (1999) Genetic control of hormone-induced ovulation in mice. Biol Reprod 61:851-856

Sudo S, Kudo M, Wada S, Sato O, Hsueh AJ, Fujimoto S (2002) Genetic and functional analyses of polymorphisms in the human FSH receptor gene. Mol Hum Reprod 8:893-839

Tinkanen H, Blauer M, Laippala P, Tuohimaa P, Kujansuu E (1999) Prognostic factors in controlled ovarian hyperstimulation. Fertil Steril 72:932-936

Ulloa-Aguirre A, Midgley AR Jr, Beitins IZ, Padmanabhan V (1995) Follicle-stimulating isohormones: characterization and physiological relevance. Endocr Rev 16:765-787

Zambrano E, Zarinan T, Olivares A, Barrios-de-Tomasi J, Ulloa-Aguirre A (1999) Receptor binding activity and in vitro biological activity of the human FSH charge isoforms as disclosed by heterologous and homologous assay systems: implication for the structure-function relationship of the FSH variants. Endocrine 10:113-121 